\title{
Functional Differential Equations and Age Dependent Population Growth*
}

\author{
N. HARRIS MCCLAMROCH \\ Computer, Information, and Control Engineering, \\ The University of Michigan, Ann Arbor, Michigan
}

Communicated by Richard Bellman

\begin{abstract}
Growth equations are established for a population of individuals that have fixed age dependent reproduction and mortality rates. Equations are obtained both for the population density and for the numerical size of the population in a fixed age group. Age and time dependent migration is taken into consideration. The usual integral equation of renewal type for these variables is shown to be equivalent to a functional differential cquation of retarded type; these differential equations are of main interest in this work. The role of initial data in characterizing a unique solution of the functional differential equation is examined in detail. Finally, some special cases for the reproduction and mortality rates are considered where the functional differential equations take a reasonably simple form.
\end{abstract}

\section{INTRODUCTION}

Processes involving the numerical growth of objects that are selfreproducing and subject to failure occur in a number of different contexts. An interesting case is where the reproduction (birth) rate and the survivorship (mortality) rate are dependent on the age of the individual objects. Properties of interest are the age distribution and the size of the population of objects. In this work mathematical models of such age dependent processes are carefully examined. The objectives are: (1) to formulate age dependent models in terms of functional differential equations, (2) to take into account initial conditions at $t=0$, i.e. representations of the process previous to $t=0$ that uniquely characterize the age distribution and size of the population after $t=0,(3)$ to distinguish between the age density function and the number of individuals in an age group, and (4) to introduce age dependent migration. Although special classes of age dependent

* This research was supported entirely by the National Science Foundation under Grant No. GK 5798.

Copyright (C) 1972 by American Elsevier Publishing Company, Inc. 
processes have been studied in great detail, basic questions concerning a broader formulation of such processes have generally been ignored. The motivation for this work arises from the area of mathematical demography [II], from which much of the terminology is derived; the ideas should also be applicable to certain problems in ecology [15], biology [14], economics [2], and industrial maintenance [1, 12].

The basic equation which usually occurs in the study of age dependent processes is the renewal equation [4] which is an integral equation for an age density function, typically the density of individuals of age zero. Under the usual assumptions concerning the reproduction and survivorship functions the renewal equation is shown to have a differentiated form, which can be expressed as a functional differential equation of retarded type [8]. In addition to consideration of the age density function the numerical size of the population in a fixed age group is also considered. In fact, this variable is shown to satisfy the same functional differential equation as that satisfied by the age density function, but with different initial conditions. Next, the effects of age dependent migration are examined; the resulting functional differential equations are obtained and the influence of initial conditions for the migration process is examined. Finally, a number of special cases are considered; the resulting functional differential equations take some particularly simple forms.

In this work only continuous time and continuous age models are examined. Discrete time and discrete age models can be obtained from our models, as in Ref. 6, by replacing derivatives by the appropriate differences and integrals by summations. One of the disadvantages of the discrete model, from a conceptual standpoint, is that one is necessarily concerned with approximating the process between the specified time points by use of some numerical integration scheme; such difficulty does not occur in the continuous model. The process is also assumed to be deterministic. Some stochastic models have been considered for age dependent processes, as by Kendall [10], but the models are generally so complex as to yield little insight into the process. Finally, the reproduction and survivorship rates are assumed to be age dependent but time independent; these rates are also assumed to be independent of the population variables. Since in this work the objective is to formulate certain models these last assumptions could have been relaxed. These assumptions were included, however, in order to concentrate primarily on the age dependent effects; extensions of the models presented here should not be difficult.

\section{PROCESSES CLOSED TO MIGRATION}

In this section equations which describe the age distribution and size of a single homogeneous population of individual objects that are closed 
to migration are derived. The models are formulated both in terms of integral equations and functional differential equations.

A quantity of interest is the density of individuals of age $s$ at time $t$, which is denoted by $x(s, t)$. For an infinitesimal age increment $d s$, the number of individuals between the ages $s$ and $s+d s$ is $x(s, t) d s$. The vital parameters are the reproduction function $b(s)$ and the survivorship function $S(s)$. The number of individuals of age zero (births) due to an individual of age between $s$ and $s+d s$ is given by $b(s) d s$. $S(s)$ denotes the proportion of individuals of age zero that survive to at least age $s$. The dimensions of $x(s, t)$ are $1 /$ time, of $b(s)$ are $1 /$ time, and $S(s)$ is dimensionless. The age $s$ and the time $t$ are measured in the same units. The functions $b(s)$ and $S(s)$ are assumed independent of time $t$ and the population is assumed, at this point, to be closed to migration. The only factors influencing the age distribution and size of the population are reproductive and survivorship factors. Although the approach is not standard, it is convenient to use the survivorship function instead of a death density function; use of the survivorship function is both simpler and somewhat more general. When it is possible to define a death density function $d(s)$, sometimes called the force of mortality, then

$$
S(s)=\exp \left\{-\int_{0}^{s} d(u) d u\right\}, \quad 0 \leqslant s<\infty .
$$

Note that only nonnegative ages are considered. It is clear that the reproduction and survivorship functions should be nonnegative for all allowable ages, i.e.

$$
\begin{aligned}
& b(s) \geqslant 0, \quad s \geqslant 0, \\
& S(s) \geqslant 0, \quad s \geqslant 0 .
\end{aligned}
$$

The reproduction function $b(s)$ is also assumed to be a function of bounded variation (locally) on $0 \leqslant s<\infty$ and right continuous on that interval. The survivorship function $S(s)$ satisfies $S(0)=1$ and is monotcnically nonincreasing; hence it is of bounded variation (locally) on $0 \leqslant s<\infty$; $S(s)$ is also assumed to be right continuous. This completes the assumptions which are required. Note that the only assumptions not based on physical arguments are the technical continuity assumptions on $b(s)$ and $S(s)$. Now consider, mathematically, the relationship between the variables which have been introduced. It is convenient, for the original derivations, to assume that $-\infty<t<\infty$; this assumption is relaxed shortly.

Using the definition of the reproduction function $b(s)$ and assuming that the population is homogeneous the density of individuals of age zero is given by

$$
x(0, t)=\int_{0}^{\infty} b(s) x(s, t) d s, \quad-\infty<t<\infty .
$$


Using the definition of the survivorship function the density of individuals of age $u$ at time $t$ consists only of individuals of age $s$ at time $t+s-u$ that survive from age $s$ to age $u$. Then

$x(u, t)=\frac{S(u)}{S(s)} x(s, t+s-u), \quad 0 \leqslant u<\infty \quad, \quad-\infty<t<\infty$.

The following convention is made: if $0 \leqslant u \leqslant s$ then $S(s)>0$ is explicitly assumed, if $0 \leqslant s \leqslant u$ then $S(s)=0$ necessarily implies that $S(u)=0$ and define $0 / 0=0$. Equations 1 and 2 are the two basic equations in this section.

A single equation for the population density function at the fixed age $\xi$ can be obtained. Assume that $\xi \geqslant 0$ is a fixed age such that $S(\xi)>0$; continue to write $x(\xi, t)$ even though $\xi$ is fixed. Substituting Eq. 2 into Eq. 1, simplifying and letting $s=\xi$, a single equation for the population density of age $\xi$ results

$$
x(\xi, t)=\int_{-\infty}^{t} b(t-u) S(t-u) x(\xi, u) d u, \quad-\infty<t<\infty .
$$

Notice that if $x(\xi, t)$ is known for $-\infty<t<\infty$ then $x(s, t)$ can be determined for all $0 \leqslant s<\infty,-\infty<t<\infty$, from Eq. 2. Note also that the form of Eq. 3 is invariant with respect to the particular age $\xi$.

Equation 3 is an integral equation for $x(\xi, 1)$; it is basically the equation studied in Refs. 4, 6, and 11 . Under the stated assumptions on $b(s)$ and $S(s)$ it is shown in the Appendix that $x(\xi, t)$ is differentiable as a function of time $t$; it is not, however, necessarily differentiable as a function of the age variable $\xi$. Differentiating Eq. 3, as indicated in the Appendix, a functional differential equation is obtained for the age density function

$$
\frac{d x}{d t}(\xi, t)=b(0) x(\xi, t)-\int_{-\infty}^{t} x(\xi, u) d_{u}\{b(t-u) S(t-u)\},
$$

Equation 4 basically represents a balance between the rate of change of the density function and the rate at which past values of the age density function contribute to the current time rate of change. Of particular interest in the above is the special case where $\xi=0$.

The previous equations have been derived based on the stated assumptions being valid for $-\infty<t<\infty$. Although the derivation of Eq. 4 was straightforward it should be clear that there are an infinite number of solutions of Eq. 4, i.e. functions which satisfy Eq. 4 identically, on $-\infty<t<\infty$. A useful mathematical model should have the property that it has a unique solution.

To obtain a unique solution consider Eq. 4 only on the interval $0<t<\infty$, together with a description of initial data at $t=0$ which summarizes the age density function $x(\xi, t)$ previous to $t=0$. In terms of 
Eq. 4 information is required which summarizes $x(\xi, t)$ for $-\infty<t \leqslant 0$ in the sense that there is a unique solution of Eq. 4 on the interval $0<t<$ $\infty$. The initial time is taken arbitrarily as $t=0$, without loss of generality. It should be emphasized that there are innumerable ways of describing the initial data for the age density function; only the simplest and most interesting descriptions are considered here.

In order to make clear what information summarizes the age density function previous to $t=0 \mathrm{Eq} .4$ can be rewritten for $0<t<\infty$ as

$$
\begin{aligned}
\frac{d x}{d t}(\zeta, t) & =b(0) x(\xi, t)-\int_{0}^{t} x(\xi, u) d_{u}\{b(t-u) S(t-u)\} \\
- & \int_{-\infty}^{0} x(\xi, u) d_{u}\{b(t-u) S(t-u)\}, \quad 0<t<\infty .
\end{aligned}
$$

From the theory of functional differential equations $[7,8]$ it follows that if the quantities

$$
\begin{gathered}
-\int_{-\infty}^{0} x(\xi, u) d_{u}\{b(t-u) S(t-u)\}, \quad 0<t<\infty, \\
x(\xi, 0),
\end{gathered}
$$

are known functions there is a unique age density function which is a solution of Eq. 4 on the interval $0<t<\infty$. In this sense it is clear that knowledge of the age density function $\tilde{x}(\xi, t),-\infty<t \leqslant 0$, is sufficient to determine

$$
\begin{gathered}
-\int_{-\infty}^{0} x(\xi, u) d_{u}\{b(t-u) S(t-u)\}=\int_{0}^{\infty} \tilde{x}(\xi,-u) d_{u}\{b(t+u) S(t+u)\}, \\
x(\xi, 0)=\tilde{x}(\xi, 0)=\int_{0}^{\infty} b(u) S(u) \tilde{x}(\xi,-u) d u,
\end{gathered}
$$

and hence a unique solution of Eq. 4 on $0<t<\infty$. To distinguish information previous to $t=0$ the tilde notation is used. Thus Eq. 4 can be rewritten on the restricted interval $0<t<\infty$, taking into account the initial conditions, as

$$
\begin{aligned}
\frac{d x}{d t}(\xi, t)= & b(0) x(\xi, t)-\int_{0}^{t} x(\xi, u) d_{u}\{b(t-u) S(t-u)\} \\
& \quad+\int_{0}^{\infty} \tilde{x}(\xi,-u) d_{u}\{b(t+u) S(t+u)\}, \quad 0<t<\infty, \\
x(\xi, 0)= & \tilde{x}(\xi, 0)
\end{aligned}
$$

Note that an alternate expression for $x(\xi, 0)$ in Eq. 5 is

$$
x(\xi, 0)=\int_{0}^{\infty} b(u) S(u) \tilde{x}(\xi,-u) d u .
$$


These two expressions for $x(\xi, 0)$ are clearly equivalent if $\tilde{x}(\xi, t)$ satisfies Eq. 3 on $-\infty<t \leqslant 0$. In summary, it follows that knowledge of $\tilde{x}(\xi, t),-\infty<t \leqslant 0$, determines a unique solution of Eq. 5 on $0<t<$ $\infty$, and hence a unique $x(s, t), 0 \leqslant s<\infty, 0<t<\infty$. Note that the initial data $\tilde{x}(\xi, t),-\infty<t \leqslant 0$, must be specified so that the indicated indefinite integral is absolutely convergent. This type of assumption is required throughout.

There are other ways of describing the initial data. Equation 5 is expressed in terms of the age density function $\tilde{x}(\xi, t),-\infty<t \leqslant 0$, at the fixed age $\xi$. It is possible to summarize the age densiiy function previous to $t=0$ in terms of the age density function at the fixed time $t=0$. By substituting Eqs. 2 and 3 and simplifying, we obtain

$$
\begin{aligned}
& -\int_{-\infty}^{0} x(\xi, u) d_{u}\{b(t-u) S(t-u)\} \\
& =S(\xi) \int_{\xi}^{\infty} \frac{\tilde{x}(u, 0)}{S(u)} d_{u}\{b(t+u-\xi) S(t+u-\xi)\}, \quad 0<t<\infty, \\
& \quad x(\xi, 0)=\tilde{x}(\xi, 0)=S(\xi) \int_{\xi}^{\infty} \frac{b(u-\xi) S(u-\xi)}{S(u)} \tilde{x}(u, 0) d u .
\end{aligned}
$$

Hence Eq. 4 can also be rewritten on $0<t<\infty$ as

$$
\begin{aligned}
\frac{d x}{d t}(\xi, t)= & b(0) x(\xi, t)-\int_{0}^{t} x(\xi, u) d_{u}\{b(t-u) S(t-u)\} \\
& +S(\xi) \int_{\xi}^{\infty} \frac{\tilde{x}(u, 0)}{S(u)} d_{u}\{b(t+u-\xi) S(t+u-\xi)\}, \quad 0<t<\infty, \\
x(\xi, 0) & =\tilde{x}(\xi, 0),
\end{aligned}
$$

which necessarily has a unique solution for $0<t<\infty$ for each function $\tilde{x}(s, 0), \xi \leqslant s<\infty$. An alternate characterization for $x(\xi, 0)$ in Eq. 6 is given by

$$
x(\xi, 0)=S(\xi) \int_{\xi}^{\infty} \frac{b(u-\xi) S(u-\xi)}{S(u)} \tilde{x}(u, 0) d u .
$$

The above two expressions for $x(\xi, 0)$ are equivalent if $\tilde{x}(s, 0), \xi \leqslant s<\infty$, is compatible with Eqs. 1 and 2.

Note that in each of Eqs. 5 and 6 the current time rate of change of the age density function depends on the sum of the rates of change due to individuals in the population previous to $t=0$ and due to individuals that have been reproduced since $t=0$.

The above two ways of characterizing the initial conditions for the age density function are only illustrative; they are not exhaustive. For example, if $0 \leqslant \xi^{\prime} \leqslant \xi^{\prime \prime}$ are fixed then $\tilde{x}(s, 0), 0 \leqslant s \leqslant \xi^{\prime \prime}$, and $\tilde{x}\left(s,-\xi^{\prime}\right), \xi^{\prime \prime}-\xi^{\prime} \leqslant$ $s<\infty$, also can be used as initial data. Appropriate functional differential 
equations can be derived which characterize a unique $x(\xi, t)$ on $0<t<\infty$ in terms of this data.

Equations have been derived which describe the age density function $x(\xi, t)$ at a fixed age $\xi$. It is often convenient to examine a dimensionless population variable such as the number of individuals in a fixed age group. For convenience only, consideration is given to the number of individuals between ages zero and the fixed age $n$ at time $t$, which is denoted by $X(n, t)$, so that

$$
X(n, t)=\int_{0}^{n} x(s, t) d s, \quad-\infty<t<\infty .
$$

In order to obtain equations solely in terms of $X(n, t)$ Eqs. 3 and 4 can be integrated with respect to the age variable to obtain the integral equation

$$
X(n, t)=\int_{-\infty}^{t} b(t-u) S(t-u) X(n, u) d u, \quad-\infty<t<\infty .
$$

It follows, as indicated in the Appendix, that $X(n, t)$ is differentiable as a function of the time $t$ and satisfies

$$
\begin{aligned}
\frac{d X}{d t}(n, t) & =b(0) X(n, t) \\
& -\int_{-\infty}^{t} X(n, u) d_{u}\{b(t-u) S(t-u)\}, \quad-\infty<t<\infty .
\end{aligned}
$$

Note that Eqs. 8 and 9 are in the same form as Eqs. 3 and 4 for the density functions.

As before, a unique solution for Eq. 9 on $0<t<\infty$ can be obtained by summarizing $X(n, t)$ previous to $t=0$. Using Eq. 9 and proceeding as in the derivation of Eq. 5 it follows that

$$
\begin{aligned}
\frac{d X}{d t}(n, t)= & b(0) X(n, t)-\int_{0}^{t} X(n, u) d_{u}\{b(t-u) S(t-u)\} \\
& +\int_{0}^{\infty} \tilde{X}(n,-u) d_{u}\{b(t+u) S(t+u)\}, \quad 0<t<\infty, \\
X(n, 0)= & \tilde{X}(n, 0),
\end{aligned}
$$

or alternately in Eq. 10

$$
X(n, 0)=\int_{0}^{\infty} b(u) S(u) \tilde{X}(n,-u) d u
$$

so that knowledge of $\widetilde{X}(n, t),-\infty<t \leqslant 0$, uniquely determines $X(n, t)$, $0<t<\infty$. Note that knowledge of $\tilde{x}(s, t), 0 \leqslant s \leqslant n,-\infty<t \leqslant 0$, allows determination of $\tilde{X}(n, t),-\infty<t \leqslant 0$, and hence uniquely determines $X(n, t), 0<t<\infty$. 
In terms of the density of individuals at the fixed time $t=0$ it follows by integrating Eq. 6 with respect to the age variable that

$$
\begin{aligned}
\frac{d X}{d t}(n, t)= & b(0) X(n, t)-\int_{0}^{t} X(n, u) d_{u}\{b(t-u) S(t-u)\} \\
& +\int_{0}^{\infty}\left\{\int_{u}^{n+u} \frac{S(s-u)}{S(s)} \tilde{x}(s, 0) d s\right\} d_{u}\{b(t+u) S(t+u)\}, \\
X(n, 0)= & \int_{0}^{n} \tilde{x}(s, 0) d s,
\end{aligned}
$$

so that knowledge of $\tilde{x}(s, 0), 0<s<\infty$ uniquely determines $\tilde{X}(n, t)$, $0<t<\infty$. An alternate expression for $X(n, 0)$ in Eq. 11 is

$$
X(n, 0)=\int_{0}^{n}\left\{\int_{0}^{\infty} \frac{b(s) S(s)}{S(s+u)} \tilde{x}(s+u, 0) d s\right\} S(u) d u .
$$

The above represent the simplest charactcrizations of the initial data. Other characterizations can easily be obtained. Of particular interest is the special case where $n=\infty$ since $X(\infty, t)$ is the total number of individuals in the population of any age.

\section{PROCESSES OPEN TO MIGRATION}

With few exceptions models which have been used to describe age dependent processes have not allowed for migration either into or out of the population of interest. In this section the previous developments are modified to include the effects of age dependent and time varying migration. Functional differential equations for the age density function and the population size, where migration is allowed, are obtained. The role of initial data in summarizing both the native population and the migration process previous to $t=0$ is examined.

Both age density functions and functions representing the numerical size of the population are introduced. In the remaining part of this work let $x(s, t) d s$ denote the number of individuals in the population, not including migrants in the population, that are between ages $s$ and $s+d s$ at time $t ; x(s, t) d s$ consists only of native individuals. Let the number of individuals in the population, taking into account migrants, that are between the ages $s$ and $s+d s$ at time $t$ be given by $z(s, t) d s$. Finally let $X(n, t)$ denote the number of individuals, not including migrants, that are between ages zero and $n$ at time $t$; let $Z(n, t)$ denote the number of individuals, including migrants, that are between ages zero and $n$ at time $t$.

The migration variables are defined as follows. The migration age density rate $v(s, t)$ is defined so that $v(s, t) d s d t$ denotes the number of migrants that enter or leave the basic population during the time interval 
$t$ to $t+d t$ that are between the ages $s$ and $s+d s$. The corresponding migration age density function is introduced where $m(s, t) d s$ denotes the number of migrants at time $t$ that are betwcen ages $s$ and $s+d s$. The migration functions are allowed to be either positive, corresponding to net immigration into the basic population, or negative, corresponding to a net emigration from the basic population. Note that the quantity $v(s, t)$ is a density rate and has dimensions (1/time) $)^{2}$ while $x(s, t), z(s, t)$ and $m(s, t)$ are density functions of dimensions $1 /$ time. The quantities $X(n, t)$ and $Z(n, t)$ are dimensionless.

The following relations should be clear from the above definitions

$$
\begin{aligned}
x(s, t) & =z(s, t)-m(s, t), \quad 0 \leqslant s<\infty, \quad-\infty<t<\infty, \\
X(n, t) & =\int_{0}^{n} x(s, t) d s, \quad 0 \leqslant n<\infty, \quad-\infty<t<\infty, \\
Z(n, t) & =\int_{0}^{n} z(s, t) d s, \quad 0 \leqslant n<\infty, \quad-\infty<t<\infty,
\end{aligned}
$$

so that

$$
X(n, t)=Z(n, t)-\int_{0}^{n} m(s, t) d s, \quad 0 \leqslant n<\infty, \quad-\infty<t<\infty .
$$

The reproduction function $b(s)$ and the survivorship function $S(s)$ are as defined previously and apply to the total population including migrants. The usual assumptions are made regarding $b(s)$ and $S(s)$.

The dynamic relations between the basic variables are now presented. Since the population has homogeneous reproduction rate the density of individuals of age zero, which are necessarily in the native population, is given by

$x(0, t)=\int_{0}^{\infty} b(s) x(s, t) d s+\int_{0}^{\infty} b(s) m(s, t) d s, \quad-\infty<t<\infty$.

The native population density of age $u$ at time $t$ consists of the density of individuals of age $s$ at time $t+s-u$ that survive from age $s$ to age $u$. Thus

$$
x(u, t)=\frac{S(u)}{S(s)} x(s, t+s-u), \quad 0 \leqslant u<\infty, \quad-\infty<t<\infty .
$$

From Eq. 12 it also follows that

and

$$
z(0, t)=\int_{0}^{\infty} b(s) z(s, t) d s, \quad-\infty<t<\infty,
$$

$$
\begin{gathered}
z(u, t)=\frac{S(u)}{S(s)}[z(s, t+s-u)-m(s, t+s-u)]+m(u, t), \\
0 \leqslant u<\infty, \quad 0 \leqslant s<\infty, \quad-\infty<t<\infty .
\end{gathered}
$$


It is possible to relate the rate at which migrants enter or leave the population to the age density of the migrants. The migrant of age $s$ at time $t$ consist of individuals that migrated between times $t-s$ and time $t$ that survive to time $t$. Hence

$$
\begin{array}{r}
m(s, t)=\int_{t-s}^{t} \frac{S(s)}{S(s-t+u)} V(s-t+u, u) d u, \\
0 \leqslant s<\infty, \quad-\infty<t<\infty .
\end{array}
$$

As in the previous section the age density functions are first examined and functional differential equations are obtained; then the equations describing the numerical size of the population are derived. In each case emphasis is placed on summarizing the process previous to $t=0$ in order to characterize a unique solution after $t=0$.

An integral equation for the age density function $x(\xi, t)$ is obtained by substituting Eq. 17 into Eq. 16 and simplifying to obtain

$$
\begin{aligned}
x(\xi, t)=\int_{-\infty}^{t} b(t-u) S(t-u) x(\xi, u) d u \\
\quad+S(\xi) \int_{0}^{\infty} b(u) m(u, t-\xi) d u, \quad-\infty<t<\infty .
\end{aligned}
$$

Using Eq. 12, Eq. 21 can be written in terms of the density function $z(\xi, t)$ as

$$
\begin{aligned}
z(\xi, t)= & \int_{-\infty}^{t} b(t-u) S(t-u) z(\xi, u) d u \\
& +m(\xi, t)-\int_{-\infty}^{t} b(t-u) S(t-u) m(\xi, u) d u \\
& +S(\xi) \int_{0}^{\infty} b(u) m(u, t-\xi) d u, \quad-\infty<t<\infty .
\end{aligned}
$$

It is possible to develop the succeeding equations in terms of the density functions $x(\xi, t)$ or $z(\xi, t)$, but it is convenient, and in fact no less general, to consider here only the case $\xi=0$. Thus the equations to follow are expressed in terms of the density of individuals of age zero.

Letting $\xi=0$ in Eq. 21 and making use of Eq. 20 leads to

$$
\begin{aligned}
x(0, t)=\int_{-\infty}^{t} b(t-u) S(t-u) x(0, u) d u & \\
& +\int_{0}^{\infty} b(u) m(u, t) d u, \quad-\infty<t<\infty,
\end{aligned}
$$

or in terms of the migration age density rate 


$$
\begin{aligned}
x(0, t)= & \int_{-\infty}^{t} b(t-u) S(t-u) x(0, u) d u \\
& +\int_{-\infty}^{t} \int_{0}^{\infty} \frac{b(s+t-u) S(s+t-u)}{S(s)} v(s, u) d s d u,
\end{aligned}
$$

Under the stated assumptions it follows, as indicated in the Appendix, that $x(0, t)$ is differentiable and

$$
\begin{aligned}
& \frac{d x}{d t}(0, t)=b(0) x(0, t)-\int_{-\infty}^{t} x(0, u) d_{u}\{b(t-u) S(t-u)\} \\
& +\int_{0}^{\infty} b(u) v(u, t) d u+\int_{-\infty}^{t} \int_{0}^{\infty} \frac{v(s, u)}{S(s)} d_{s}\{b(s+t-u) S(s+t-u)\} d u \\
& -\infty<t<\infty
\end{aligned}
$$

The above equation expresses the fact that the time rate of change of the density of individuals of age zero consists of a component due to the inherent dynamic characteristics of the reproduction and survivorship functions and a component due to the reproduction of migrants.

Even for a known migration policy it is clear that Eq. 23 possesses an infinite number of solutions on $-\infty<t<\infty$. In order to characterize a unique solution on the interval $0<t<\infty$ consideration of the process previous to $t=0$ is required. Throughout, the migration process $v(s, t)$, $0<s<\infty, 0<t<\infty$, is assumed known. The question arises as to what information sufficiently summarizes the process previous to $t=0$ so that there is a unique age density function and $0<t<\infty$. The results of the previous section indicated several ways in which the history of $x(0, t)$ previous to $t=0$ could be summarized. In this section consideration is also given to summarizing the migration process previous to $t=0$. There are many ways of summarizing the migration process previous to $t=0$; only the simplest and most interesting ways are considered.

Proceeding as in the previous section Eq. 23 can be rewritten as

$$
\begin{aligned}
\frac{d x}{d t}(0, t)= & b(0) x(0, t)-\int_{0}^{t} x(0, u) d_{u}\{b(t-u) S(t-u)\} \\
& -\int_{-\infty}^{0} x(0, u) d_{u}\{b(t-u) S(t-u)\}+\int_{0}^{\infty} b(u) v(u, t) d u \\
& +\int_{0}^{t} \int_{0}^{\infty} \frac{v(s, u)}{S(s)} d_{s}\{b(s+t-u) S(s+t-u)\} d u \\
& +\int_{-\infty}^{0} \int_{0}^{\infty} \frac{v(s, u)}{S(s)} d_{s}\{b(s+t-u) S(s+t-u)\} d u, \\
& -\infty<t<\infty,
\end{aligned}
$$


and it follows that if in addition to $v(s, t), 0<s<\infty, 0<t<\infty$, the quantities

$$
\begin{gathered}
-\int_{-\infty}^{0} x(0, u) d_{u}\{b(t-u) S(t-u)\}, \quad 0<t<\infty, \\
\int_{-\infty}^{0} \int_{0}^{\infty} \frac{v(s, u)}{S(s)} d_{s}\{b(s+t-u) S(s+t-u)\} d u, \quad 0<t<\infty, \\
x(0,0)
\end{gathered}
$$

are known there is a unique solution on $0<t<\infty$. Using the tilde notation to denote quantities previous to $t=0$ obtain

$$
\begin{gathered}
-\int_{-\infty}^{0} x(0, u) d_{u}\{b(t-u) S(t-u)\}= \\
\int_{0}^{\infty} \tilde{x}(0,-u) d_{u}\{b(t+u) S(t+u)\} \\
\int_{-\infty}^{0} \int_{0}^{\infty} \frac{v(s, u)}{S(s)} d s\{b(s+t-u) S(s+t-u)\} d u \\
=-\int_{0}^{\infty} \int_{0}^{\infty} \frac{\tilde{v}(s,-u)}{S(s)} d_{s}\{b(s+t+u) S(s+t+u)\} d u \\
x(0,0)=\tilde{x}(0,0)=\int_{0}^{\infty} b(u) S(u) \tilde{x}(0,-u) d u \\
+\int_{0}^{\infty} \int_{0}^{\infty} \frac{b(s+u) S(s+u)}{S(s)} \tilde{v}(s,-u) d s d u .
\end{gathered}
$$

Hence a functional differential equation for $x(0, t)$ is

$$
\begin{aligned}
& \frac{d x}{d t}(0, t)=b(0) x(0, t)-\int_{0}^{t} x(0, u) d_{u}\{b(t-u) S(t-u)\} \\
& +\int_{0}^{\infty} \tilde{x}(0,-u) d u\{b(t+u) S(t+u)\}+\int_{0}^{\infty} b(u) v(u, t) d u \\
& +\int_{0}^{t} \int_{0}^{\infty} \frac{v(s, u)}{S(s)} d_{s}\{b(s+t-u) S(s+t-u)\} d u \\
& -\int_{0}^{\infty} \int_{0}^{\infty} \frac{\tilde{s}(s,-u)}{S(s)} d_{s}\{b(s+t+u) S(s+t+u)\} d u, \quad 0<t<\infty \\
& x(0,0)=\tilde{x}(0,0) .
\end{aligned}
$$

An alternative expression for $x(0,0)$ in Eq. 24 is given by

$$
\begin{aligned}
x(0,0)= & \int_{0}^{\infty} b(u) S(u) \tilde{x}(0,-u) d u \\
& +\int_{0}^{\infty} \int_{0}^{\infty} \frac{b(s+u) S(s+u)}{S(s)} \tilde{v}(s,-u) d s d u .
\end{aligned}
$$


In summary, for a known migration policy $v(s, t), 0<s<\infty, 0<t<\infty$, the quantities $\tilde{x}(0, t),-\infty<t \leqslant 0, \tilde{v}(s, t), 0<s<\infty,-\infty<t<0$, determine a unique solution of Eq. 24 on the interval $0<t<\infty$. The age density function for the total population, including migrants, can be determined using Eqs. 12 and 20 to be

$$
\begin{aligned}
& z(s, t)= S(s) \tilde{x}(0, t-s)+\int_{0}^{s-t} \frac{S(s)}{S(s-t-u)} \tilde{v}(s-t-u,-u) d u \\
&+\int_{0}^{t} \frac{S(s)}{S(s-t+u)} v(s-t+u, u) d u, \quad 0 \leqslant t<s, \\
& z(s, t)= S(s) x(0, t-s)+\int_{t-s}^{t} \frac{S(s)}{S(s-t+u)} v(s-t+u, u) d u, \\
& s \leqslant t<\infty .
\end{aligned}
$$

A similar equation can be developed for $x(s, t)$.

Now consider another representation of the process previous to $t=0$. Using Eqs. 18-20 obtain

$$
\begin{gathered}
-\int_{-\infty}^{0} x(0, u) d_{u}\{b(t-u) S(t-u)\} \\
+\int_{-\infty}^{0} \int_{0}^{\infty} \frac{v(s, u)}{S(s)} d_{s}\{b(s+t-u) S(s+t-u)\} d u \\
=\int_{0}^{\infty} \frac{\tilde{z}(u, 0)}{S(u)} d_{u}\{b(t+u) S(t+u)\}, \\
x(0,0)=\tilde{z}(0,0)=\int_{0}^{\infty} b(u) \tilde{z}(u, 0) d u .
\end{gathered}
$$

Hence from Eq. 23

$$
\begin{aligned}
& \frac{d x}{d t}(0, t)=b(0) x(0, t)-\int_{0}^{t} x(0, u) d_{u}\{b(t-u) S(t-u)\} \\
&+ \int_{0}^{\infty} \frac{\tilde{z}(u, 0)}{S(u)} d_{u}\{b(t+u) S(t+u)\}+\int_{0}^{\infty} b(u) v(u, t) d u \\
&+ \int_{0}^{t} \int_{0}^{\infty} \frac{v(s, u)}{S(s)} d_{s}\{b(s+t-u) S(s+t-u)\} d u, \\
& 0<y<\infty,
\end{aligned}
$$

$x(0,0)=\tilde{z}(0,0)$.

An alternate representation for $x(0,0)$ in Eq. 26 is

$$
x(0,0)=\int_{0}^{\infty} b(u) \tilde{z}(u, 0) d u .
$$


Thus for known $v(s, t), 0<s<\infty, 0<t<\infty$, the quantities $\check{z}(s, 0)$, $0 \leqslant s<\infty$, determine a unique solution of Eq. 26 on the interval $0<t<$ $\infty$. From Eq. 12 it follows that $\tilde{x}(s, 0), 0 \leqslant s<\infty$, and $\tilde{m}(s, 0), 0 \leqslant s<$ $\infty$, determine $\tilde{z}(s, 0), 0 \leqslant s<\infty$; hence they also determine a unique solution of Eq. 26. Using Eqs. 19 and 20 the age density function for the population, including migrants, is determined to be

$$
\begin{gathered}
z(s, t)=\frac{S(s)}{S(s-t)} \tilde{z}(s-t, 0)+\int_{0}^{t} \frac{S(s)}{S(s-t+u)} v(s-t+u, u) d u, \\
0 \leqslant t<s, \\
z(s, t)=S(s) x(0, t-s)+\int_{t-s}^{t} \overline{S(s-t+u)} v(s-t+u, u) d u, \\
s \leqslant t<\infty .
\end{gathered}
$$

A similar equation can be developed for $x(s, t)$.

Functional differential equations have been obtained for the density of individuals of age zero where migration has been allowed. Two representations of the process previous to $t=0$ have been presented; these two representations are only illustrative of a whole class of ways of summarizing the process previous to $t=0$. In addition, equations have been given for $z(s, t)$ in terms of $x(0, t)$ and the appropriate description of the process previous to $t=0$. As mentioned earlier it is possible to obtain explicit functional differential equations for $x(s, t)$ and $z(s, t)$ although the resulting equations are rather lengthy.

Attention now turns to consideration of the number of individuals between ages zero and the fixed age $n$ at time $t$. By integrating Eq. 21 with respect to the age variable, we obtain the integral equation:

$$
\begin{aligned}
X(n, t)=\int_{-\infty}^{t} b(t-u) S(t-u) X(n, u) d u \\
\quad+\int_{0}^{n} \int_{0}^{\infty} S(s) b(u) m(u, t-s) d u d s, \quad-\infty<t<\infty,
\end{aligned}
$$

for the number of individuals, not including migrants, between the ages zero and $n$ at time $t$. Similarly, an integral equation can be obtained for the total number of individuals between ages zero and $n$, including migrants,

$$
\begin{array}{rl}
Z(n, t)=\int_{-\infty}^{t} & b(t-u) S(t-u) Z(n, u) d u \\
& +\int_{0}^{n} \int_{0}^{\infty} S(s) b(u) m(u, t-s) d u d s+\int_{0}^{n} m(s, t) d s \\
& -\int_{-\infty}^{t} \int_{0}^{n} b(t-u) S(t-u) m(s, u) d s d u, \quad-\infty<t<\infty .
\end{array}
$$


Although functional differential equations can be obtained for $X(n, t)$ and $Z(n, t)$, in the general case the resulting equations become rather lengthy. Hence, for purposes of illustration only, a special but important case is considered; functional differential equations for the total native population $Z(\infty, t)$ are obtained. Equations are also obtained which relate the total population, including migrants, $Z(\infty, t)$, to the variable $X(\infty, t)$ and the initial data.

Hence from Eq. 28 we obtain

$$
\begin{aligned}
& X(\infty, t)=\int_{-\infty}^{t} b(t-u) S(t-u) X(\infty, u) d u \\
&+ \int_{-\infty}^{t} S(t-u)\left\{\int_{0}^{\infty} b(s) m(s, u) d s\right\} d u, \\
&-\infty<t<\infty,
\end{aligned}
$$

or its equivalent functional differential equation form

$$
\begin{aligned}
& \frac{d X}{d t}(\infty, t)= b(0) X(\infty, t)-\int_{-\infty}^{t} X(\infty, u) d_{u t}\{b(t-u) S(t-u)\} \\
&+\int_{0}^{\infty} b(s) m(s, t) d s-\int_{-\infty}^{t}\left\{\int_{0}^{\infty} b(s) m(s, u) d s\right\} d_{u} S(t-u) \\
&-\infty<t<\infty
\end{aligned}
$$

The first two terms on the right hand side of Eq. 30 represent the time rate of change of the native population due to reproduction and mortality of the native population itself, the third term represents the rate of increase due to reproduction of migrants, while the last term represents the rate of decrease of the native population due to mortality of offspring of migrants. In terms of the migration density rate Eq. 20 can be used to obtain

$$
\begin{aligned}
\frac{d X}{d t}(\infty, t) & =b(0) X(\infty, t)-\int_{-\infty}^{t} X(\infty, u) d_{u}\{b(t-u) S(t-u)\} \\
& +\int_{-\infty}^{t} \int_{0}^{\infty} c(t, s, u) \frac{v(s, u)}{S(s)} d s d u, \quad-\infty<t<\infty,
\end{aligned}
$$

where

$$
\begin{aligned}
c(t, s, u)= & b(s+t-u) S(s+t-u) \\
& -\int_{u}^{t} b(s+\lambda-u) S(s+\lambda-u) d_{\lambda} S(t-\lambda), \\
& \quad 0 \leqslant s<\infty, \quad 0 \leqslant u \leqslant t<\infty .
\end{aligned}
$$

In order to characterize a unique $X(\infty, t), 0<t<\infty$, it is possible to proceed as before to obtain 


$$
\begin{aligned}
& \frac{d X}{d t}(\infty, t)= b(0) X(\infty, t)-\int_{0}^{t} X(\infty, u) d_{u}\{b(t-u) S(t-u)\} \\
&+\int_{0}^{\infty} \tilde{X}(\infty,-u) d_{u}\{b(t+u) S(t+u)\} \\
&+\int_{0}^{t} \int_{0}^{\infty} c(t, s, u) \frac{v(s, u)}{S(s)} d s d u \\
&+\int_{0}^{\infty} \int_{0}^{\infty} c(t, s,-u) \frac{\tilde{v}(s,-u)}{S(s)} d s d u, \quad 0<t<\infty \\
& X(\infty, 0)=\tilde{X}(\infty, 0) .
\end{aligned}
$$

An alternate form for $X(\infty, 0)$ in Eq. 32 is

$$
\begin{aligned}
X(\infty, 0)= & \int_{0}^{\infty} b(u) S(u) \tilde{X}(\infty,-u) d u \\
& +\int_{0}^{\infty} \int_{0}^{\infty}\left\{\int_{0}^{u} S(\lambda) b(s+u-\lambda) S(s+u-\lambda) d \lambda\right\} \frac{\tilde{v}(s,-u)}{S(s)} d s d u .
\end{aligned}
$$

Thus for a known migration policy $v(s, t), 0<s<\infty, 0<t<\infty$, $\tilde{X}(\infty, t),-\infty<t \leqslant 0$, and $\tilde{v}(s, t), 0<s<\infty,-\infty<t<0$, the data determine a unique solution of Eq. 32 on $0<t<\infty$. The number of individuals, including migrants, is determined using Eq. 15 to be

$$
\begin{aligned}
z(\infty, t)=X(\infty, t) & +\int_{0}^{\infty} \int_{0}^{\infty} \frac{S(s+t+u)}{S(s)} \tilde{v}(s,-u) d s d u \\
+ & \int_{0}^{t} \int_{0}^{\infty} \frac{S(s+t+u)}{S(s)} v(s, u) d s d u, \quad 0<t<\infty
\end{aligned}
$$

Another way of describing the process previous to $t=0$ is in terms of data at the fixed time $t=0$. Using Eqs. 13 and 17, it follows that

$$
\begin{aligned}
& \frac{d X}{d t}(\infty, t)=b(0) X(\infty, t)-\int_{0}^{t} X(\infty, u) d_{u}\{b(t-u) S(t-u)\} \\
&+\int_{0}^{\infty}\left\{\int_{0}^{s} S(s-u) d_{u}[b(t+u) S(t+u)]\right\} \frac{\tilde{x}(s, 0)}{S(s)} d s \\
&+\int_{0}^{\infty} \int_{0}^{\infty} b(s) \tilde{m}(s,-u) d s d_{u} S(t+u) \\
&+\int_{0}^{\infty} c(t, s, 0) \frac{\tilde{m}(s, 0)}{S(s)} d s \\
&+\int_{0}^{t} \int_{0}^{\infty} c(t, s, u) \frac{v(s, u)}{S(s)} d s d u, \quad 0<t<\infty, \\
& X(\infty, 0)=\int_{0}^{\infty} \tilde{x}(s, 0) d s .
\end{aligned}
$$


An alternate expression for $X(\infty, 0)$ in Eq. 34 is

$$
\begin{aligned}
X(\infty, 0)=\int_{0}^{\infty}\left\{\int_{0}^{\infty} \frac{b(s) S(s)}{S(s+u)} \tilde{x}(s+u, 0) d s\right\} S(u) d u \\
+\int_{0}^{\infty} \int_{0}^{\infty} S(u) b(s) \tilde{m}(s,-u) d s d u .
\end{aligned}
$$

For a known migration policy $v(s, t), 0<s<\infty, 0<t<\infty$, the quantities $\tilde{x}(s, 0), 0 \leqslant s<\infty$, and $\tilde{m}(s, t), 0 \leqslant s<\infty,-\infty<t \leqslant 0$, determine a unique solution of Eq. 34 on the interval $0<t<\infty$. The total number of individuals, including migrants, is given by

$$
\begin{aligned}
Z(\infty, t)=X(\infty, t) & +\int_{0}^{t} \int_{0}^{\infty} \frac{S(s+t-u)}{S(s)} v(s, u) d s d u \\
& +\int_{0}^{\infty} \frac{S(s+t)}{S(s)} \tilde{m}(s, 0) d s, \quad 0<t<\infty .
\end{aligned}
$$

Functional differential equations have been developed for the number of native individuals $X(\infty, t)$; two representations of the process previous to $t=0$ have been presented. As expected, the net migration $\int_{0}^{\infty} m(s, t) d s$ is generally not sufficient to determine $X(\infty, t)$ or $Z(\infty, t)$; knowledge of the age structure of the migration process is required. As indicated earlier, functional differential equations for $X(n, t)$ and for $Z(n, t)$ can be developed using the procedures indicated.

\section{SOME SPECIAL FORMS FOR REPRODUCTION AND SURVIVORSHIP FUNCTIONS}

In the previous sections, concern has focused on the development of functional differential equations for describing age dependent processes, for general reproduction and survivorship functions. In this section some special cases of these functions are considered and the resulting functional differential equations are shown to assume some rather simple forms. A number of these special forms have been used as models for growth processes in the literature but usually without a clear understanding of the implicitly assumed underlying age structure of the process. Hence, one of the contributions of this work is to relate some of the simple functional differential equations to their underlying assumptions about the age structure. In the examples to follow the functional differential equations are expressed in terms of an age density function at $t=0$. Such description of the initial data is probably of most interest. 
Before considering some special cases in detail mention is made of the class of so-called branching processes where

$$
\begin{aligned}
& b(s)=2 d(s), \quad 0 \leqslant s<\infty, \\
& S(s)=\exp \left\{-\int_{0}^{s} d(r) d r\right\}, \quad 0 \leqslant s<\infty .
\end{aligned}
$$

Since the reproduction rate is twice the mortality rate, one interpretation of the above is that if an individual dies then it is replaced, through the reproduction process, by two individuals of age zero. Such processes have been extensively studied $[9,13,19]$ using integral equations; it should be clear that branching processes can also be studied in terms of a functional differential equation formulation as devcloped in the preceding sections. To the author's knowledge such an approach has not been taken.

Now return to consideration of some special forms for the reproduction and survivorship functions. First, suppose that the reproduction rate is a constant and the survivorship function is exponential, i.e. the force of mortality is constant; then

Then from Eq. 22

$$
\begin{aligned}
& b(s)=b, \quad 0 \leqslant s<\infty, \\
& S(s)=e^{-d s,} \quad 0 \leqslant s<\infty .
\end{aligned}
$$

$$
x(0, t)=\int_{-\infty}^{t} b e^{-d(t-u)} x(0, u) d u+\int_{-\infty}^{t} \int_{0}^{\infty} b e^{-d(t-u)} v(s, u) d s d u,
$$

and from Eq. 23

$$
\begin{aligned}
\frac{d x}{d t}(0, t)=b x & (0, t)-\int_{-\infty}^{t} d b e^{-d(t-t)} x(0, u) d u \\
& +\int_{0}^{\infty} b v(s, t) d t+\int_{-\infty}^{t} \int_{0}^{\infty} d b e^{-d(t-u)} v(s, u) d s d u
\end{aligned}
$$

Substituting the first equation into the second and simplifying yields

$$
\frac{d x}{d t}(0, t)=(b-d) x(0, t)+b \int_{0}^{\infty} v(s, t) d s .
$$

The required initial condition is simply

$$
x(0,0)=\tilde{z}(0,0) .
$$

The above equation is a simple ordinary differential equation and has been used in a number of contexts $[2,11,14,15]$ to describe age dependent processes. The age density function for the total population, including migrants, is easily obtained from Eq. 27 as

$$
\begin{array}{ll}
z(s, t)=e^{-d t} \tilde{z}(s-t, 0)+\int_{0}^{t} e^{-d(t-u)} v(s-t+u, u) d u, & 0 \leqslant t<s, \\
z(s, t)=e^{-d s} x(0, t-s)+\int_{t-s}^{t} e^{-d(t-u)} v(s-t+u, u) d u, & s \leqslant t<\infty .
\end{array}
$$


Note that knowledge of $\tilde{z}(0,0)$ and the total migration $\int_{0}^{\infty} v(s, t) d s$ is sufficient to determine the density of individuals of age zero but if the density of individuals at other ages is desired then the age structure of $\tilde{z}(s, 0), v(s, t)$, is required. Using Eqs. 20,29 and 30 the size of the native population can be shown to satisfy

$$
\begin{aligned}
\frac{d X}{d t}(\infty, t)=(b & -d) X(\infty, t)+b e^{-d t} \int_{0}^{\infty} \tilde{m}(s, 0) d s \\
& +b e^{-d t} \int_{0}^{t} \int_{0}^{\infty} e^{d u} v(s, u) d s d u, \quad 0<t<\infty,
\end{aligned}
$$

$X(\infty, 0)=\int_{0}^{\infty} \tilde{x}(s, 0) d s$,

and the total size of the population, including migrants, is obtained from Eq. 35 as

$$
\begin{aligned}
Z(\infty, t)=X(\infty, t)+ & \int_{0}^{t} \int_{0}^{\infty} e^{-d(t-u)} v(s, u) d s d u \\
& +e^{-d t} \int_{0}^{\infty} \check{m}(s, 0) d s, \quad 0<t<\infty .
\end{aligned}
$$

Note that the specific age structure of the native and migrant populations at $t=0$ is not required in order to determine the total population variables. Also note that the above differential equations have simple interpretations in terms of the effects of the native population itself, the migrants in the population at time $t=0$ and the migrants that enter or leave the population after $t=0$. An even more specialized, but still interesting, case is when the reproduction rate $b=0$. After some simple computations the density of individuals, including migrants, is

$$
\begin{gathered}
z(s, t)=e^{-d t} \tilde{z}(s-t, 0)+\int_{0}^{t} e^{-d(t-u)} v(s-t+u, u) d u, \\
0 \leqslant t<s, \\
z(s, t)=e^{-d t} \tilde{z}(0,0)+\int_{t-s}^{t} e^{-d(t-u)} v(s-t+u, u) d u, \\
\quad s \leqslant t<\infty,
\end{gathered}
$$

and the total population, including migrants, is

$$
\begin{aligned}
Z(\infty, t)=e^{-d t} & \left\{\int_{0}^{\infty} \tilde{x}(s, 0) d s+\int_{0}^{\infty} \tilde{m}(s, 0) d s\right\} \\
& +\int_{0}^{t} \int_{0}^{\infty} e^{-u(t-u)} v(s, u) d s d u, \quad 0<t<\infty .
\end{aligned}
$$


Clearly the population consists only of individuals in the population at $t=0$ plus individuals that migrated after $t=0$. Also of interest is the case where the mortality rate $d=0$. Then the density of individuals, including migrants, is

$$
\begin{aligned}
z(s, t) & \tilde{z}(s-t, 0)+\int_{0}^{t} v(s-t+u, u) d u, \quad 0 \leqslant t<s, \\
z(s, t) & =e^{b(t-s)} \tilde{z}(0,0)+\int_{t-s}^{t} v(s-t+u, u) d u \\
& +b \int_{0}^{t-s} e^{b(t-s-u)} \int_{0}^{\infty} v(s, u) d s d u, \quad s \leqslant t<\infty,
\end{aligned}
$$

and the total population, including migrants, is

$$
\begin{aligned}
Z(\infty, t)=e^{b t}\left\{\int_{0}^{\infty} \tilde{x}(s, 0) d s+\int_{0}^{\infty} \tilde{m}(s, 0) d s\right\} \\
+\int_{0}^{t} \int_{0}^{\infty}\left[e^{b(t-u)}-1\right] v(s, u) d s d u, \quad 0<t<\infty .
\end{aligned}
$$

Now consider the slightly more complicated case where reproduction occurs over a finite age interval and there is a maximum age for individuals; then

$$
\begin{aligned}
b(s) & =b, & 0 & \leqslant s<T_{1} \\
& =0, & T_{1} & \leqslant s<\infty \\
S(s) & =e^{-d s}, & 0 & \leqslant s<T_{2} \\
& =0, & T_{2} & \leqslant s<\infty,
\end{aligned}
$$

where $0<T_{1} \leqslant T_{2}$. Without loss of generality assume that $\tilde{x}(s, 0)=0$, $T_{2} \leqslant s<\infty, \tilde{m}(s, 0)=0, T_{2} \leqslant s<\infty$, and $v(s, t)=0, T_{2}<s<\infty$, $0<t<\infty$. The equation for the density of individuals of age zero is determined from Eqs. 22 and 23 to be

$$
\begin{aligned}
& \frac{d x}{d t}(0, t)=(b-d) x(0, t)-b e^{-d t} \tilde{z}\left(T_{1}-t, 0\right) \\
& \quad+b \int_{0}^{T_{1}} v(s, t) d S-b \int_{0}^{t} e^{-d(t-u)} v\left(u-t+T_{1}, u\right) d u, \\
& \begin{aligned}
\frac{d x}{d t}(0, t)= & (b-d) x(0, t)-b e^{-d T_{1}} x\left(0, t-T_{1}\right) \\
& +b \int_{0}^{T_{1}} v(s, t) d s-b \int_{t-T_{1}}^{t} e^{-d(t-u)} v\left(u-t+T_{1}, u\right) d u, \\
x(0,0)=\tilde{z}(0,0) . & \left.T_{1} \leqslant t<\infty,\right]
\end{aligned}
\end{aligned}
$$


The equations for the age density function are

$$
\begin{gathered}
z(s, t)=e^{-d t} \tilde{z}(s-t, 0)+\int_{0}^{t} e^{-d(t-u)} v(s-t+u, u) d u, \\
0 \leqslant s<T_{2}, \quad 0 \leqslant t<s, \\
z(s, t)=e^{-d s} x(0, t-s)+\int_{t-s}^{t} e^{-d(t-u)} v(s-t+u, u) d u, \\
\quad 0 \leqslant s<T_{2}, \quad s \leqslant t<\infty \\
z(s, t)=0, \quad T_{2} \leqslant s<\infty, \quad 0 \leqslant t<\infty .
\end{gathered}
$$

The above are referred to as differential difference equations. Such equations have been considered $[3,16]$ as models for age dependent processes.

Note that the maximum age $T_{2}$ does not affect the equation for $x(0, t)$ since individuals between ages $T_{1}$ and $T_{2}$ do not reproduce. The quantity $\tilde{z}(s, 0), 0 \leqslant s<T_{2}$, clearly serves as initial data for determination of $x(0, t)$ and $z(s, t)$.

The equations for the native population $X(\infty, t)$ are obtained using Eqs. 20, 29, and 30 as

$$
\begin{aligned}
\frac{d X}{d t}(\infty, t)= & (b-d) X(\infty, t)-b e^{-d t} \int_{T_{1}-t}^{T_{1}+T_{2}-t} \tilde{x}(s, 0) d s \\
& +b \int_{0}^{t} \int_{0}^{T_{1}-t+u} e^{-d(t-u)} v(s, u) d s d u+b e^{-d t} \int_{0}^{T_{1}-t} \tilde{n}(s, 0) d s \\
& -b e^{-d T_{2}} \int_{0}^{T_{1}} \tilde{m}\left(s, t-T_{2}\right) d s, \quad 0 \leqslant t<T_{1},
\end{aligned}
$$

$$
\begin{aligned}
\frac{d X}{d t}(\infty, t)= & (b-d) X(\infty, t)-b e^{-d T_{1}} X\left(\infty, t-T_{1}\right) \\
& +b \int_{t-T_{1}}^{t} \int_{0}^{T_{1}-t+u} e^{-d(t-u)} v(s, u) d s d u \\
& -b e^{-d T_{2}} \int_{0}^{T_{1}} \tilde{m}\left(s, t-T_{2}\right) d s, \quad T_{1} \leqslant t<T_{2},
\end{aligned}
$$

$$
\begin{aligned}
\frac{d X}{d t}(\infty, t)= & (b-d) X(\infty, t)-b e^{-d T_{1}} X\left(\infty, t-T_{1}\right) \\
& +b \int_{t-T_{1}}^{t} \int_{0}^{T_{1}-t+u} e^{-d(t-u)} v(s, u) d s d u \\
& -b \int_{0}^{t-T_{2}} \int_{0}^{T_{1}-t+u} e^{-d(t-u)} v(s, u) d s d u \\
& -b e^{-d t} \int_{0}^{T_{1}+T_{2}-t} \tilde{m}(s, 0) d s, \quad T_{2} \leqslant t<T_{1}+T_{2}
\end{aligned}
$$




$$
\begin{aligned}
\frac{d X}{d t}(\infty, t)= & (b-d) X(\infty, t)-b e^{-d T_{1}} X\left(\infty, t-T_{1}\right) \\
& +b \int_{t-T_{1}}^{t} \int_{0}^{T_{1}-t+u} e^{-d(t-u)} v(s, u) d s d u \\
& -b \int_{t-T_{1}-T_{2}}^{t-T_{2}} \int_{0}^{T_{1}-t+u} e^{-d(t-u)} v(s, u) d s d u, \\
X(\infty, 0)= & \int_{0}^{T_{2}} \tilde{x}(s, 0) d s . T_{2} \leqslant t<\infty,
\end{aligned}
$$

The total size of the population, including migrants, as determined from Eq. 35 is

$$
\begin{array}{r}
Z(\infty, t)=X(\infty, t)+\int_{0}^{t} \int_{0}^{T_{2}-t+u} e^{-d(t-u)} v(s, u) d s d u \\
+e^{-d t} \int_{0}^{T_{2}-t} \tilde{m}(s, 0) d s, \quad 0 \leqslant t<T_{2}, \\
Z(\infty, t)=X(\infty, t)+\int_{t-T_{2}}^{t} \int_{0}^{T_{2}-t+u} e^{-d(t-u)} v(s, u) d s d u, \\
T_{2} \leqslant t<\infty .
\end{array}
$$

Assuming that $v(s, t), 0<s<T_{2}, 0<t<\infty$, is known, then $\tilde{x}(s, 0)$, $0 \leqslant s<T_{2}$ and $\tilde{m}(s, t), 0 \leqslant s<T_{2},-T_{2} \leqslant t \leqslant 0$, uniquely determine $X(\infty, t), 0<t<\infty$, and $Z(\infty, t), 0<t<\infty$. Interpretation of the above differential difference equations can easily be given in terms of the contribution to the time rate of change due to the population at $t=0$, due to the population past $t=0$, due to migrants before and at $t=0$, and due to migration past $t=0$.

The previous examples are only illustrative of the kinds of assumptions about the reproduction and survivorship functions which lead to reasonably simple forms for the various functional differential equations. Other special forms for these functions lead to specific functional differential equations which can be determined using the approach indicated.

\section{CONCLUSIONS}

Functional differential equations have been derived for the age density function and for the numerical size of the population; the effects of age dependent migration have been included. Emphasis has been placed on the role of initial data in summarizing the process previous to $t=0$, in terms of characterizing a unique solution of the functional differential equation on $0<t<\infty$. The fact that there are alternate ways of representing the initial data should have important practical implications. For 
example, it would seem reasonable to formulate a mathematical model for an age dependent process which is based on initial data which is most accessible or most accurate. The importance of the initial data has generally been overlooked in the literature on age dependent processes. It should be mentioned that there is no need for the description of the initial data to be compatible with the appropriate functional differential equations on the interval $-\infty<t \leqslant 0$, if the solution is of interest only on the interval $0<t<\infty$. In fact, the equations which take into account initial data can be derived by explicit consideration of an initial population; this is the approach usually taken in the references previously cited. It is felt that the approach used in this work which began by considering the case $-\infty<t<\infty$ and then proceeding to the case $0<t<\infty$ is simpler.

Recall that each of the functional differential equations considered are derived from an integral equation. This integral or renewal equation has received most attention in the literature on age dependent processes $[4,6,9,11]$. A more recent formulation of age dependent processes using partial differential equations is due to von Foerster [18]; such an approach has been developed in some detail by Trucco [17]. However, it is felt that added insight is given to the dynamical character of age dependent processes by using functional differential equations. There are further advantages in this approach. In either an integral or partial differential equation formulation much of the mathematical structure of the age dependent process is obscured. Since there is a reasonably well-developed theory of functional differential equations $[7,8]$ this mathematical structure should be recognized so that it can be used to advantage. Secondly, there are definite practical advantages of using functional differential equations in terms of computer simulation. Finally, as indicated by some special cases, the underlying dynamic character of some age dependent processes may be rather simple when the process is formulated in terms of functional differential equations.

In the preceding development the following assumptions are made. The reproduction and survivorship functions are age dependent but time independent; the reproduction and survivorship processes are linear functions of the population process; only a single homogeneous population of individuals is considered. Each of these assumptions could be relaxed so that models of age dependent processes could be developed for multiple population groups whose reproduction and survivorship functions are nonlinear and time varying. Fredrickson [5] has examined such a problem using von Foerster's equation. Clearly the models indicated in this work are a necessary first step in developing a more general theory for age dependent processes based on the use of functional differential equations. 
This work has been concerned solely with developing models for age dependent processes in terms of functional differential equations. The usefulness of these models ultimately depends on the analysis of the behavior of solutions of such equations. Solution properties of the functional differential equations which occur in this work have not been presented here; this subject is to be examined in subsequent work. However, mention should be made of two general areas of some practical interest for which the present formulation of age dependent processes should be particularly appropriate. One area is the study of asymptotic properties of age dependent processes under various fixed migration policies. A second area is the study of the relationship between a migration policy, considered as a control, in terms of the succeeding effects on both the population density and the size of the population. These subject areas certainly deserve additional study; the formulation of age dependent processes in terms of functional differential equations should allow a direct investigation of such subjects.

\section{APPENDIX}

It has been asserted that certain integral equations are equivalent to functional differential equations under the stated assumptions. To mathematically justify these statements, the following theorem is presented.

\section{THEOREM}

Let $h(s), 0 \leqslant s<\infty$, and $k(s), 0 \leqslant s<\infty$, be known functions of bounded variation and let $g(t),-\infty<t<\infty$, be a known integrable function. Then each solution $x(t),-\infty<t<\infty$, of the integral equation

$$
\begin{aligned}
x(t)=\int_{-\infty}^{t} h(t-s) x(s) d s & \\
& +\int_{-\infty}^{t} k(t-s) g(s) d s, \quad-\infty<t<\infty,
\end{aligned}
$$

is a solution of the functional differential equation

$$
\begin{aligned}
\frac{d x}{d t}(t) & =h(0) x(t)-\int_{-\infty}^{t} x(u) d_{u} h(t-u) \\
& +k(0) g(t)-\int_{-\infty}^{t} g(u) d_{u} k(t-u), \quad-\infty<t-\infty,
\end{aligned}
$$

and vice versa.

Proof

For convenience define the function

$$
\begin{aligned}
\tilde{h}(s) & =h(s)-h(0), \quad 0 \leqslant s<\infty \\
& =0, \quad-\infty<s<0 .
\end{aligned}
$$


Consider the following string of equalities.

$$
\begin{aligned}
\int_{-\infty}^{t}\left\{h(0) x(s)-\int_{-\infty}^{s} x(u) d u h(s-u)\right\} d s \\
\quad=\int_{-\infty}^{t} x(u) h(0) d u-\int_{-\infty}^{t} \int_{-\infty}^{s} x(u) d_{u} \tilde{h}(s-u) d s \\
=\int_{-\infty}^{t} x(u) h(0) d u-\int_{-\infty}^{t} \int_{-\infty}^{t} x(u) d_{u} \tilde{h}(s-u) d s \\
=\int_{-\infty}^{t} x(u) h(0) d u-\int_{-\infty}^{t} x(u) d_{u}\left\{\int_{-\infty}^{t} \tilde{h}(s-u) d s\right\} \\
=\int_{-\infty}^{t} x(u) h(0) d u-\int_{-\infty}^{t} x(u) d u\left\{\int_{u}^{t} \tilde{h}(s-u) d s\right\} \\
=\int_{-\infty}^{t} x(u) h(0) d u-\int_{-\infty}^{t} x(u) d_{u}\left\{\int_{0}^{t-u} \tilde{h}(s) d s\right\} \\
=\int_{-\infty}^{t} x(u) h(0) d u+\int_{-\infty}^{t} x(u) \tilde{h}(t-u) d u \\
=\int_{-\infty}^{t} x(u) h(t-u) d u, \quad-\infty<t<\infty .
\end{aligned}
$$

In the above use has been made of the definition of $\tilde{h}(s)$ and a Fubini theorem due to Cameron and Martin. Hence

$$
\begin{aligned}
& \int_{-\infty}^{t}\left\{h(0) x(s)-\int_{-\infty}^{s} x(u) d_{u} h(s-u)\right\} d s \\
&=\int_{-\infty}^{t} h(t-s) x(s) d s, \quad-\infty<t<\infty .
\end{aligned}
$$

Since the left hand side is obviously differentiable so is the right hand side of the equation. Hence from Eq. A, the term $x(t)-\int_{-\infty}^{t} h(t-s) g(s) d s$ is differentiable. A similar argument to the above yields that

$$
\begin{aligned}
& \int_{-\infty}^{t}\left\{k(0) g(s)-\int_{-\infty}^{s} g(u) d_{u} k(s-u)\right\} d s \\
&=\int_{-\infty}^{t} k(t-s) g(s) d s, \quad-\infty<t<\infty .
\end{aligned}
$$

hence $\int_{-\infty}^{t} k(t-s) g(s) d s$ is differentiable and thus so is $x(t)$. Using formulas $C$ and $D$ the equivalence of Eqs. A and B follows directly. 


\section{REFERENCES}

1 R. E. Barlow and F. Proschan, Mathematical Theory of Reliability, Wiley, New York (1965).

2 E. Burmeister and A. R. Dobell, Mathematical Theories of Economic Growth, Macmillan, New York (1970).

3 K. L. Cooke, in Mathematical Theory of Control. Academic Press, New York (1967).

4 W. Feller, Ann. Math. Stat. 12, 243 (1941).

5 A. G. Fredrickson, Mathematical Biosciences 10, 117 (1971).

6 L. A. Goodman, J. Royal Stat. Soc. A80, 541 (1967).

7 A. Halanay, Differential Equations, Academic, New York (1966).

8 J. Hale, Functional Differential Equations, Springer-Verlag, New York (1971).

9 T. E. Harris, The Theory of Branching Processes, Springer-Verlag, New York (1963).

10 D. G. Kendall, J. Royal Stat. Soc. B11, 230 (1949).

11 N. Kcyfitz, Introduction to the Mathematics of Population, Addison-Wesley, Reading, Mass. (1968).

12 A. J. Lotka, Ann. Math. Stat. 10, 1 (1939).

13 H. M. Martinez, Bull. Math. Biophys. 28411 (1966).

14 J. H. Milsum, Biological Control Systems Analysis, McGraw-Hill, New York (1966).

15 E. C. Pielou, An Introduction to Mathematical Ecology, Wiley-Interscience, New York (1969).

16 K. P. Tognetti and A. Mazanov, Mathematical Biosciences 8, 355 (1970).

17 E. Trucco, Bull. Math. Biophys, 27, 285, 449 (1965).

$18 \mathrm{H}$. Von Foerster, in The Kinetics of Cellular Proliferation, Grune and Stratton, New York (1959).

19 W. A. O'N. Waugh, Biometrika 42, 291 (1955). 